\title{
La influencia del internet y su impacto en los adolescentes
}

The influence of the internet and its impact on adolescents.

\author{
Pablo Homero Velasteguí. ${ }^{1}$
}

\section{Resumen.}

Si bien sabemos la tecnología ha dado pasos agigantados en la actualidad, si recordamos como era en tiempos antiguos podemos notar los grandes cambios que han ido surgiendo gracias a la innovación constante que palpamos. La sociedad día a día está más con la tecnología, y es que nos hemos ido adaptando a todo aquello que ha surgido para beneficio nuestro, para agilizar tareas, las empresas gracias a los avances tecnológicos llevan su negocio más a la cumbre mencionando como un avance el internet pues en el podemos hacer cuanto podamos.

En el ámbito empresarial gracias al internet y ahora en la actualidad en muchas aplicaciones o de hecho al navegar encontramos muchos anuncios de empresas, de productos, entre otros, con la finalidad de poder dar a conocer a los usuarios los servicios que poseen las empresas, todo esto ha hecho posible surgir.

La tecnología si bien sabemos ha dado grandes cambios en beneficio de la sociedad, pero esta a su vez perjudica a los jóvenes porque hace que estén más pegados a ella y sientan la necesidad de estar siempre conectados a Internet ya sea enviando mensajes, viendo videos o a su vez gastando el tiempo en los videojuegos hasta cierto punto lo llamamos beneficioso, pero abusar de las tecnologías ya no es correcto por eso hay que tener un poco de concientización del tiempo y el uso que le estamos dando a estar en Internet.

Los jóvenes con facilidad se enredan en el mundo de la tecnología como ejemplo tenemos los celulares siempre quieren tener el más reciente y quieren mantenerse al día con la tecnología, pero de uno dependerá si queremos que esto nos cause una adicción o controlarlo.

Palabras claves: TIC, adolescente, uso patológico de internet, móvil, videojuegos, adicción, adicciones conductuales, focalización, psicología, trastornos, compulsivos.

\footnotetext{
${ }^{1}$ Ciencia digital, Ambato, Ecuador, luisefrainvelastegui@ cienciadigital.org
} 


\section{Abstract.}

While we know that technology has made giant leaps in the present, if we remember how it was in ancient times we can notice the great changes that have been emerging thanks to the constant innovation that we feel. The society day by day is more with technology, and that is that we have adapted to everything that has emerged for our benefit, to streamline tasks, companies thanks to technological advances take their business more to the top, mentioning it as an advance the internet because in it we can do everything we can.

In the business world thanks to the internet and nowadays in many applications or in fact when browsing we find many announcements of companies, of products, among others, in order to be able to inform users about the services that companies have, all this has made it possible to arise.

Although technology knows we have made great changes for the benefit of society, but this in turn harms young people because it makes them more attached to it and feel the need to be always connected to the Internet either by sending messages, watching videos or We spend time in videogames to a certain extent, we call it beneficial, but abusing technology is no longer correct, so we must have a little awareness of the time and the use we are making of being on the Internet.

Young people are easily entangled in the world of technology as an example we have cell phones always want to have the latest and want to keep up with technology, but one will depend if we want this to cause an addiction or control it.

Keywords: ICT, adolescent, pathological use of internet, mobile, videogames, addiction, behavioral addictions, focalization, psychology, disorders, compulsive.

\section{Introducción}

\section{La influencia del internet como impacto influyente en los adolescentes.}

Las tecnologías de la información y comunicación en varias investigaciones de (Levis, 2002; Saldaña, 2001; Young, 1998) La sociedad actual se establece en un mundo cambiante, en una época de transformaciones y en un periodo de transición a la adaptación de la sociedad a las tecnologías. Internet, móviles y videojuegos ocupan un espacio importante en el proceso de socialización, influyendo en comportamientos y actitudes (Levis, 2002); en consecuencia, la psicología, como ciencia y como práctica profesional no puede permanecer ajena a esta transformación.

A lo largo del tiempo han surgido diferentes tipos de conductas debido a las Tecnologías de la Información y la Comunicación, ya que estas herramientas fueron creadas con la intención de informar, comunicar, pero esto ha ido cambiando por el mal uso que se le da a esto, y esto ha hecho que en los adolescentes se genere conductas adictivas. Las tecnologías de la información y la comunicación (TIC) han realizado grandes avances para la sociedad, esta generalmente ha influenciado más en lo jóvenes y adolescentes, esto hace que sea imprescindible realizar evaluaciones sobre los cambios y adaptaciones que se genera en los adolescentes y esto hace que se realice evaluaciones sobre el desarrollo emocional y psicosocial en los mismos. 
No hay que dejar a un lado la importancia que tienen las TIC, pero a su vez debemos ser conscientes en lo que estas tecnologías generan cambios en los adolescentes causando de cierta manera una adicción de estas mayormente en Internet, dispositivos móviles y los videojuegos. Se debería realizar criterios sobre este comportamiento que genera una adicción en los hábitos saludables de los adolescentes.

Para los autores (Black, 1999; Davis, 2001; Grohol, 2005; Machargo, 2003; Simkova, 2004) las TIC están presentes en todos los ámbitos de la realidad científica, cultural y social, y constituyen un elemento esencial del funcionamiento de nuestra vida cotidiana. La adolescencia es una etapa que merece una atención especial con respecto a sus relaciones con las TIC, sobre todo porque es un colectivo muy sensible al momento y al entorno social en el que vive y también porque estas tecnologías están especialmente presentes en su vida. Los adolescentes, fascinados por internet, el móvil y los videojuegos, han encontrado en estas tecnologías un medio extraordinario de relación, comunicación, aprendizaje, satisfacción de la curiosidad, ocio y diversión. Poco a poco, esto conlleva que las TIC se conviertan en un elemento importante e imprescindible en sus vidas.

Las TIC son un buen elemento para la sociedad gracias a su avance tecnológico que ha ayudado a muchos, pero algo que debemos mencionar es que al mismo tiempo en que la tecnología crece la juventud se sumerge en el internet pasándose horas y horas navegando, en el chat, jugando, etc. Esto genera una adicción, aunque creen que esto no es así pues no lo notan, el cambio constante que tenemos influye en nuestro comportamientos y actitudes. Estas TIC nos han servido mucho, gracias a esto se ha facilitado grandes tareas, por ejemplo, las TIC han sido implementadas en la educación, negocios etc. Siendo este un gran paso a mejoras y conocimientos tanto para los estudiantes como para los docentes, y en el ámbito empresarial ayudando tener un mejor marketing, esto y más podemos hacer con las TIC, pero los jóvenes no lo usan así.

\section{Adicciones conductuales y tecnológicas}

Las adicciones conductuales y tecnológicas en varias investigaciones de los autores (Alonso, 2003; Echeburúa, 1999; Griffiths, 1997) mencionan que el estudio de las adicciones conductuales es un fenómeno emergente por varias razones. En primer lugar, existe un interés creciente por contemplar muchas conductas como adictivas, estas conductas pueden ser tan diversas como las compra, el trabajo, el sexo y el juego de apuestas). En segundo lugar, llegar a comprender las características de las adicciones conductuales incrementa nuestro conocimiento de la psicología de las adicciones químicas. Y, en tercer lugar, la evolución tecnológica plantea un periodo de adaptación a las nuevas formas de adquirir información y comunicación que pueden generar trastornos o desadaptaciones psicológicas más o menos permanentes. Entiende por adicciones tecnológicas aquellas que involucran la interacción hombre-máquina y las divide entre pasivas (como la televisión) y activas (Internet, móvil y videojuegos).

El diagnóstico de la adicción a las TIC parte del mismo principio que las adicciones a sustancias. Se señalan tres síntomas nucleares: 
$\checkmark$ Incapacidad de control e impotencia. La conducta se realiza pese al intento de controlarla y/o no se puede detener una vez iniciada.

$\checkmark$ Dependencia psicológica. Incluye el deseo, ansia o pulsión irresistible (craying) y la polarización o focalización atencional (la actividad se convierte en la más importante al dominar pensamientos y sentimientos).

$\checkmark$ Efectos perjudiciales graves en diferentes ámbitos para la persona (conflicto intrapersonal: experimentación subjetiva de malestar) y/o su ámbito familiar y social (conflicto interpersonal: trabajo, estudio, ocio, relaciones sociales, etc.)

Los síntomas no esenciales más frecuentes son:

a) Tolerancia y abstinencia.

b) Modificación del estado de ánimo. Sensación creciente de tensión que precede inmediatamente el inicio de la conducta. Placer o alivio mientras e realiza la conducta. Agitación o irritabilidad si no es posible realizar la conducta.

c) Euforia y trance mientras se desarrolla la actividad.

d) Negación, ocultación y/o minimización.

e) Sentimientos de culpa y disminución de la autoestima.

f) Riesgo de recaída y de reinstauración de la adicción.

Los adolescentes presentan un aumento de adicción al permanecer horas y horas frente a un computador ya sea en el chat o tan solo mirando videos, si bien nosotros podemos poner un límite a esto, pero no siempre ocurre así, muchos decimos solo serán unos minutos y luego sin darnos cuenta nos hemos pasado horas en el chat.

Y a esto sumémosle la incapacidad del joven de darle un límite, y solo se conecta no importa la hora y más de lo habitual, por ejemplo, los adolescentes se quedan largas horas en el chat y más aún en la noche y no miden el tiempo que ha pasado perdiendo así las horas necesarias para descansar, esto le generaría un desorden en su salud. También cuando se sienten en depresión recurren al internet para huir de los problemas y sentirse bien a través de la red del internet.

Según (Young, 1998; Young, 1999; Wieland, 2005; Griffiths, 2000) Todo gira en torno a la realidad virtual. La red domina su vida con una total focalización atencional. Se observan a nivel escolar, efectos perjudiciales grave (fracaso escolar, abandono de estudios) y se pueden producir pequeños hurtos para compras de elementos y créditos para juegos como Habbo Hotel o Everquest. Aparecen sentimientos de culpa y disminución de la autoestima. El adolescente siente que internet es el único lugar donde puede sentiré bien, pero a la larga aparece soledad, y reducción del bienestar psicológico.

La importancia que debemos darle a este tipo de conductas atrayente de las TIC, es la razón por la que los jóvenes y adolescentes caen en una crisis cibernética ¿Por qué? Porque este medio es lo que provoca, pues se entretiene en los chats y en momentos de soledad podría hacer que el chico se refugie aquí, y no es correcto ese hábito, pero es lo que se está palpando hoy en día con la juventud. 
En este tema notamos que la tecnología se vuelve atrayente a tal grado que puede hacer que los jóvenes no puedan estar sin tener la tecnología cerca de ellos, claro ejemplo tenemos los teléfonos móviles la cual es uno de los avances tecnológicos que tenemos a nuestro alcance y con ello acceso a internet, lo que produce que los jóvenes y no solo ellos, muchos en general, no pueden estar un momento sin revisar, mensajes, correo, ver videos en internet, etc. Todo esto es considerado como algo atrayente. Ante esto podemos decir que las TIC si no le damos un correcto uso podríamos convertirnos en dependientes de ellas, afectando así nuestra mente, y como se mencionaba en el párrafo, afecta nuestro sentido del humor, y el adolescente o los jóvenes parecerían de síntomas tanto físicos como psicológicos, por ello siempre hemos escuchado que debemos darle el uso debido y no abusar ni desperdiciar el tiempo vanamente en cosas que no son tan importantes.

\section{Adolescencia y las nuevas tecnologías}

Según estudios realizados por (Castellana, 2003; Johansson, 2004; Matute, 2003) La adolescencia es un fenómeno psicológico, biológico, social y cultural. Es el periodo donde el individuo se desarrolla hacia la adquisición de la madurez psicológica a partir de construir su identidad personal. También es un periodo importante por sus propias características evolutivas: omnipotencia, tendencia a buscar la causa de sus problemas en el otro (culpar al exterior), poca experiencia de vida, dificultad de reconocer adicciones sutiles y necesidad de normalizar conductas de riesgo.

Un adolescente está en la etapa más importante de su vida porque es donde aprenderá a desarrollar su madurez y sobre todo formar su identidad personal, aunque el internet es muy bueno sabemos que no le están dando el uso adecuado que debería es por ello que el adolescente cae en un estado de dependencia hacia el internet.

Todas estas características convierten a la adolescencia en un periodo sumamente susceptible de sufrir conductas adictivas u otros trastornos psicológicos relacionados con el uso de alguna de las aplicaciones de las TIC. Un 1,98\% de una muestra de 3237 adolescentes noruegos entre 12 y 18 años cumplirían criterios de adicción y un 8,66\% presentarían un uso de alto riesgo (Ibídem, 2004).

Aunque, para (Matute, 2003, pág. 66), se trata sobre todo de usuarios inexpertos y no de posibles adictos, no se debe olvidar que la necesidad del adolescente para acceder a la comunicación e información (actualmente elevada, heterogénea y cambiante) y el hecho de que siempre son usuarios inexpertos, aumenta la vulnerabilidad de este colectivo. Mientras los usuarios experimentados dedican más tiempo al correo electrónico, actividades relacionadas con su trabajo, consulta de noticias y de información, los usuarios de noveles pasan más tiempo en chats y otros servicios de ocio.

La adolescencia se encuentra en un periodo tan susceptible de padecer conductas adictivas, esto quiere decir que no es tan difícil asociar una conducta adictiva de un adolescente con las TIC, en vez de buscar contenido educativo en beneficio de sí mismo, las personas que trabajan ocupan la tecnología como el internet para revisar correos electrónicos, actividades con relación a su empleo, etc. Los jóvenes la usan para ver videos, pasar tiempo chat en internet y en tanto servicio de ocio que proporciona la 
internet, es un tiempo mal invertido que causa en los adolescentes un desperdicio de ellos y sin beneficio. Sabemos que cada día la tecnología se desarrolla más y más y a la misma vez no solo los jóvenes, la sociedad en general se encuentra en una etapa de constante desarrollo de conocimientos, ya que si hay algo nuevo debe actualizar sus conocimientos para ser competitivo. Citemos un ejemplo de tecnología como lo es la misma internet, donde podemos pasar horas y horas navegando sin medir el tiempo que este consume y en muchas ocasiones no le estamos dando el debido uso que este debería.

\section{Internet y Adolescentes}

En la investigación realizada sobre el internet y los adolescentes destacan (Chamarro, 2005; Estallo, 1995; Madrid, 2000) Que la Internet puede definirse como una red de redes de ordenador para compartir datos y recursos. El abaratamiento de los costos de conexión, las mejoras tecnológicas y la llegada masiva de ordenadores personales a los hogares, provoca un crecimiento exponencial del número de usuarios; por este motivo, cada vez adquieren mayor importancia las respuestas de los usuarios y conviene valorar algunos efectos que la irrupción de Internet produce en sus conductas.

El uso que hace el adolescente de Internet es relevante. Las horas de conexión son distintas según la red con la tendencia de las chicas a conectare más que los chicos. Respecto al entorno de conexión, la mayoría se conecta desde sus respectivos hogares $(78,4 \%)$ de los cuales la mitad tienen su propio ordenador y línea de Internet personal, el resto se conectan desde la escuela (26\%), biblioteca (8\%) o cibercafé (5\%). A través del chat, el $32 \%$ de los menores facilitan su teléfono, el $17 \%$ ha quedado físicamente con alguien y se han conocido personalmente a través de una cita y el $34 \%$ se han sentido incomodo en la red en alguna ocasión. (Ibídem, 1995).

Una de las más grandes tecnologías es la internet donde podemos encontrar grandes cantidades de información útil para todos, a la misma vez donde podemos postear nosotros también información que le pueda ser útil a alguien más. Cientos de millones de usuarios a diario visitan y usan este medio para compartir o bajar información. Si recordamos un poco de la historia antes no todos podíamos tener acceso al internet y solo muy pocos podían hacerlo, pero ahora todos pueden hacerlo, solo hay que darle el uso que se debe y no desaprovechar el tiempo en cosas vanas.

En la investigación realizada por (Castellana, 2006; King, 1996; Fiel, 2001) dicen que respecto a los motivos que mueven al adolescente a conectarse a la red, se ha venido observando que giran en torno a la posibilidad de estar en contacto y vinculare con su grupo de iguales superando la distancia física, así como expresar y hablar de temas que desde la relación cara a cara les sería difícil o imposible de realizar. El efecto desinhibidor del anonimato y la ausencia de contacto visual le permite expresar alguna necesidad o emoción desagradable o; en otras ocasiones, ser honesto, abierto y expresar emociones sobre asuntos personales que no podrían ser fácilmente discutidos frente a frente. El adolecente se encuentra en un mundo diferente, sin las limitaciones del mundo "real", un lugar donde se oculta la vergüenza y surgen las intimidades de su mundo interno. 
En la edad de un joven sobre es donde Internet se convierte en un atractivo a la vista de ellos porque se pueden relacionar con más personas, ya sea conocidas o personas que no conoce, es aquí donde se pierde toda platica verbal y solo se disponen a hablar y hablar por vía internet, el fallo de esto es que al ser una plática virtual el joven puede esconder su verdadera personalidad, digamos que el adolescente alivia su aburrimiento, el estrés, pasando horas chat en internet.

Como ya se ha mencionado en párrafos anteriores la internet es de mucha ayuda para todos pero todo claro está depende del tipo de uso que le estemos dando nosotros, en la internet encontramos mucha información que nos ayudaría a fortalecer conocimientos, a educarnos, pero esto a la larga no siempre se da así pues lo adolescentes se han dejado llevar más por las redes sociales y el ocio que esto produce pues conlleva pasar largas horas en el chat, jugando o en alguna actividad de poca ayuda mental para el adolescente, absorbiendo tiempo que podría ser usado en reforzar conocimientos y buscando información útil para sí. Cabe mencionar que no todo lo que está en la internet es adictivo, lo que considero que es más adictivo son las redes sociales, videojuegos.

\section{Adolescentes y Móvil}

(Forunati, 2002; Ling, 2002; Sánchez, 2006) Los adolescentes se han convertido en los principales usuarios de los diferentes servicios que ofrece la telefonía móvil a los que dedican cada vez más tiempo y recursos económicos. Para esta generación, los teléfonos móviles son objetos que siempre han existido, hecho que les convierte en expertos para poder elegir el medio, lugar y el momento en que hace falta utilizar el móvil.

Realmente el éxito que ha tenido la telefonía móvil en los adolescentes ha sido muy grande ya que muchos adolescentes poseen dispositivos móviles, siempre tenemos la tecnología avanzando día a día y con ellos los móviles lo que provoca al adolescente a querer tener lo último en tecnología o tan solo para presumir para la mayoría de los jóvenes y adolescentes no ven un teléfono móvil como algo que les será útil para consultar desde un teléfono sino que lo compran para hacerse selfies, que el dispositivo tenga buena memoria que tenga buena RAM para poder jugar, es decir los adolescentes solo miran el teléfono móvil como un entretenimiento, mientras que la gente adulta lo uso más para fines laborales, ya sea guardar en el dispositivo fechas importantes para recordarlas y coordinar ese evento, la otra parte de esto tiene que ver con la cantidad de dinero que muchos invierten por un teléfono móvil,, y no son ellos quienes lo pagaran sino que con sus padres, quiñes cargan con el peso de ellos.

Un dispositivo móvil tiene acceso al internet donde pueden conectarse desde su dispositivo sin la necesidad de ir a un cyber, en el aparato, el uso principal de este dispositivo es que se creó con la intención de poder comunicarse con la familia o amigos cercanos, pero este no ha sido su uso final por completo ya que en la actualidad lo que la mayoría de personas hace con su dispositivo móvil es chat vía internet.

Las razones por la cuales el móvil es tan atractivo para los adolescentes son variadas: 
a) Tipología de la comunicación que permite el contacto continuo e inmediato con la red de relaciones sociales y expandirla.

b) Tiempo y privacidad porque el móvil es atemporal, este hecho permite conectarse con rapidez a cualquier hora del día o de la noche, así como "escapar" del control paterno.

c) Identidad personal ya que el grado de personalización que permite el móvil facilita el proceso de adquisición de la identidad personal.

d) Socialización en tanto que el móvil fomenta el proceso de emancipación de los padres y entre los adolescentes es un símbolo de Status social

e) Instrumentalidad referida al uso del aparato como despertador, reloj de bolsillo, grabadora, agenda, plataforma de juego y radio (Ibídem, 2006).

Podemos ver que la telefonía móvil de un adolescente es mas de ocio que la de un adulto, porque los jóvenes lo usan intensivamente en enviar SMS, llamadas, juegos. Este tipo de conductas que generan los mismos jóvenes no son conductas adecuadas, consumen tiempo en cosas que ni siquiera hará aumentar tu coeficiente intelectual solo hará que te estanques y en vez de leer libros o artículos para fortalecer conocimientos simplemente lo desperdiciamos estando solo de ocio.

Según (Criado, 2004; De la Gándara, 2004) El riesgo llega cuando no son capaces de desprenderse de su móvil incluso en situaciones técnicamente dificultosas, comprueban rápidamente la batería o la cobertura y además no se caracterizan tanto para la necesidad de llamar sino para sentirse receptores contantes de llamadas. Conviene tener presente, cuando se trata de adolescentes, u vulnerabilidad frente al uso del móvil, debido a que aún no tienen un control completo de sus impulsos, son más fácilmente influenciables por campañas publicitarias y han aceptado el móvil como un símbolo de status, provocando sentimientos negativos y problemas de autoestima en los que no tienen móvil o que no reciben tantas llamadas o SMS como sus compañeros.

Para las personas la adicción no es muy acertada, pero poseemos grandes cantidades de información para poder fortalecer nuestros conocimientos y poder explicar lo que incluye la adicción generada en los jóvenes y adolescentes hoy en día.

Si bien es cierto un dispositivo móvil no solo es un aparato técnico, es con lo que convivimos día tras día se convierte en algo muy imprescindible en nuestras vidas que hace que no podamos separarnos de el en ningún momento y siempre queremos revisar nuestras cuentas o redes sociales donde quiera que nos encontremos, en el teléfono móvil también encontramos hasta tips de moda actual y es así como nuestra identidad se va moldeando de acuerdo a lo que estamos mirando y al ver esta clase de publicaciones lo que ocasiona es que deseemos estar al último grito de la moda.

Los adolescentes se encuentran en una etapa donde todos quieren tener un teléfono móvil a su alcance, todos quieren estar al día con la tecnología. Así que se puede decir que se han sumergido en este mundo, si tomamos un ejemplo de un celular, muchos ven en lo que el dispositivo trae para saber si ese es el indicado para ellos, siempre buscan una buena cámara, espacio en memoria, y cualquier cosa de las que estos traen. Pero no solo 
es eso, lo que se menciona en esta parte es sobre la adicción que genera un teléfono móvil, muchos adolescentes no pueden desprenderse de sus celulares y siempre quieren estarlos manipulando, buscan una red para poder concretarse y revisar sus mensajes, entre otras cosas. Las TIC han traído una revolución con sus avances, pero no todo es positivo, porque el mal uso que le están dando provoca esta clase de adicción la cual debería controlarse y que no se convierta en algo perjudicial para el adolescente.

\section{Adolescentes y Videojuegos}

Para (Castellana, 2006; Estallo, 1995; Gee, 2004; Rodríguez, 2002) Los videojuegos son una forma de entretenimiento que no deja de crecer y hoy en día representa un negocio millonario. Miles de personas juegan con los videojuegos en sus distintas formas y tipos.

Lo que motiva al adolecente a introducirse en el mundo de los gamer es que podríamos decir que son varias, ejemplo: a muchos les gusta vivir emociones donde sienten u adrenalina e imaginan que lo que están viviendo es real, no mide consecuencias de pasar horas jugando a un videojuego, pero la verdad es que muchos videojuegos hacen que no te de ganas de desprenderte de él y quieres seguir en eso más tiempo, ahora la tecnología como la informática implementada en varios tipos como se mencionó antes si el adolescente desperdiciaba tiempo en pasar largas horas en el chat. Las pequeñas o grandes motivaciones que hacen que el adolescente practique con los videojuegos quizá el juego está muy bueno que hace que quieras seguir jugando por mucho tiempo.

Los videojuegos podemos decir que siempre ha generado controversia pues no todos estarán de acuerdo en apoyar que los videojuegos ayudan por así decirlo tu físico mental pues muchos apoyan la noción de que lo videojuegos solo consumen tu tiempo y no aportas nada a la sociedad.

Los videojuegos en un principio fueron creados con la finalidad de entretenimiento que hoy en día es un gran negocio millonario los adolescentes que lo juegan lo hacen por probar aventuras virtuales, pero esto interfiere en la conducta real del adolecente pues muchos adolescentes pasan conectados a internet y juegan por horas y se desconectan del mundo real. Aunque muchos piensan que de cierto modo los videojuegos ayudan a la destreza del joven otros opinan que interfiere en sus estudios, en la capacidad de razonar respecto a ellos, y desperdician el tiempo jugando. Pero claro todo depende del tipo de uso que le damos nosotros pues las TIC tienen fines educativos, de cierto modo, somos nosotros los que le estamos dando el mal uso a esta tecnología la cual deberíamos usar de una manera más consiente.

Con base en la Encuesta Nacional sobre Disponibilidad y Uso de Tecnologías de la Información en los Hogares (ENDUTIH) que por primera vez se levantó en 2015 para dar continuidad al Módulo precedente, al segundo trimestre se registraron 62.4 millones de personas, de seis años o más en el país, usuarias de los servicios que ofrece Internet, lo que representa el $57.4 \%$ de esta población.

Tabla N.1 


\begin{tabular}{lll}
\hline \hline Uso del internet & Frecuencia & Porcentaje \\
\hline Personas que usan internet & 62.4 & $57.4 \%$ \\
Personas que no usan & 46.3 & $42.6 \%$ \\
internet & & \\
\hline
\end{tabular}

Fuente:http://www.inegi.org.mx/saladeprensa/aproposito/2016/internet2016_0.p df

Elaborado por: Instituto Nacional de Estadística y Geografía.

\section{Gráfico N.1}

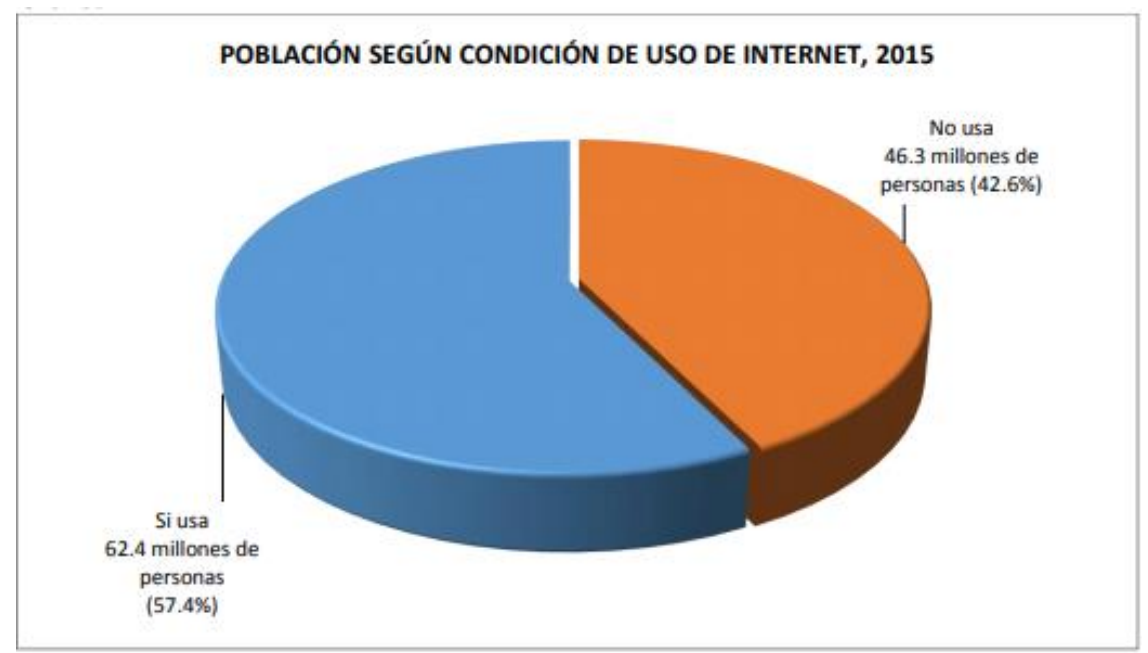

Fuente:http://www.inegi.org.mx/saladeprensa/aproposito/2016/internet2016_0.p df

Elaborado por: Instituto Nacional de Estadística y Geografía.

El acceso a esta tecnología es predominante entre los jóvenes del país. Si como se indicó anteriormente, el 57.4 por ciento del total de la población de seis años o más se declaró como usuaria de Internet, de entre los individuos de 6 a 34 años, la proporción alcanza el 73.6 por ciento, es decir que el uso de Internet es predominante en la población joven del país.

\section{Tabla N.2}

\begin{tabular}{lll}
\hline $\begin{array}{l}\text { Uso del internet por } \\
\text { edades }\end{array}$ & Si utiliza & No utiliza \\
\hline De 6 a 11 años & $53.9 \%$ & $46.1 \%$ \\
De 12 a 17 años & $85.9 \%$ & $14.1 \%$ \\
De 18 a 24 años & $83.1 \%$ & $16.9 \%$ \\
De 25 a 34 años & $71.1 \%$ & $28.9 \%$ \\
De 35 a 44 años & $56.4 \%$ & $43.6 \%$ \\
De 45 a 54 años & $41.3 \%$ & $58.7 \%$ \\
De 55 y más & $17.6 \%$ & $82.4 \%$ \\
\hline
\end{tabular}


Fuente:http://www.inegi.org.mx/saladeprensa/aproposito/2016/internet2016_0.p df

Elaborado por: Instituto Nacional de Estadística y Geografía.

\section{Gráfico N.2}

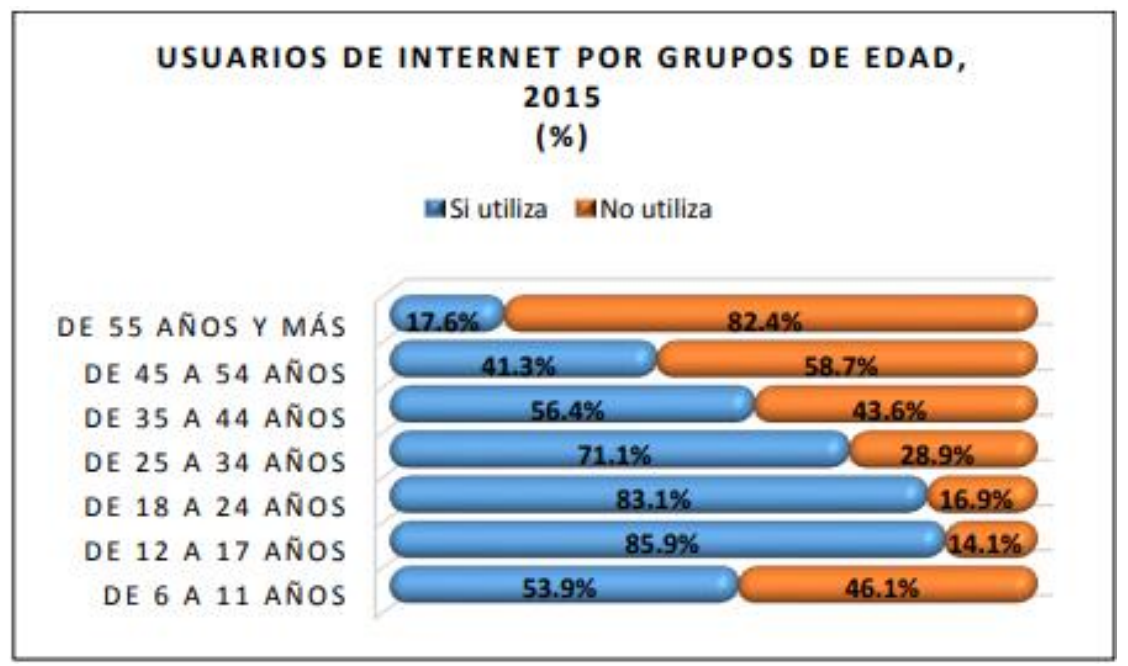

Fuente:http://www.inegi.org.mx/saladeprensa/aproposito/2016/internet2016_0.p df

Elaborado por: Instituto Nacional de Estadística y Geografía.

La gráfica 2 hace evidente que conforme aumenta la edad, decae el uso de Internet. Para el grupo de entre 35 a 44 años, poco más de la mitad (56.4 por ciento) realiza alguna actividad en la red y la proporción disminuye al 41.3 por ciento entre individuos de 45 a 54 años; solo el $17.6 \%$ de los adultos mayores a 54 años conoce y utiliza este recurso.

De la población que cuenta con estudios de nivel superior (licenciatura o posgrado), 9 de cada 10 ha incorporado el uso de Internet en sus actividades habituales; cuatro de cada cinco de los que cuentan con estudios de nivel medio superior (preparatoria o equivalente) así también lo hacen, y con nivel básico (primaria o secundaria) resultan poco menos de la mitad (46.1\%).

Tabla N.3

\begin{tabular}{lll}
\hline Uso del internet & Si utiliza & No utiliza \\
\hline Educación superior & $94.5 \%$ & $5.5 \%$ \\
Educación media & $83 \%$ & $17 \%$ \\
Educación básica & $46.1 \%$ & $53.9 \%$
\end{tabular}

Fuente:http://www.inegi.org.mx/saladeprensa/aproposito/2016/internet2016_0.p df

Elaborado por: Instituto Nacional de Estadística y Geografía.

\section{Gráfico N.3}




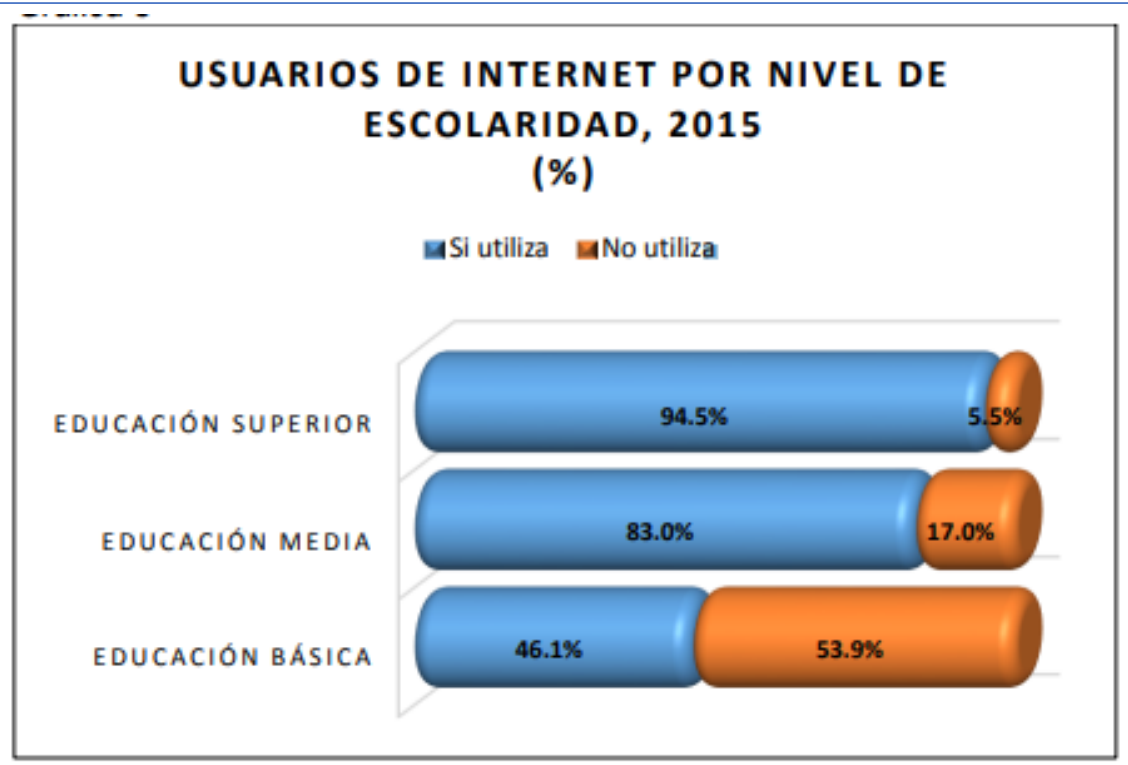

Fuente:http://www.inegi.org.mx/saladeprensa/aproposito/2016/internet2016_0.p df

Elaborado por: Instituto Nacional de Estadística y Geografía.

En la Era del Conocimiento, el acceso a Internet se encuentra asociado de manera importante con el nivel de estudios, y esto se ilustra en el gráfico 3.

En la siguiente tabla se puede observar quienes consumen más internet en el ámbito estudiantil ya sea educación superior, educación media y la educación básica.

\section{Tabla N.4}

\begin{tabular}{lll}
\hline Uso del internet & Si utiliza & No utiliza \\
\hline Educación superior & $94.5 \%$ & $5.5 \%$ \\
Educación media & $83 \%$ & $17 \%$ \\
Educación básica & $46.1 \%$ & $53.9 \%$ \\
\hline
\end{tabular}

Fuente:http://www.inegi.org.mx/saladeprensa/aproposito/2016/internet2016_0.p df

Elaborado por: Instituto Nacional de Estadística y Geografía.

\section{GRÁFICO N4}




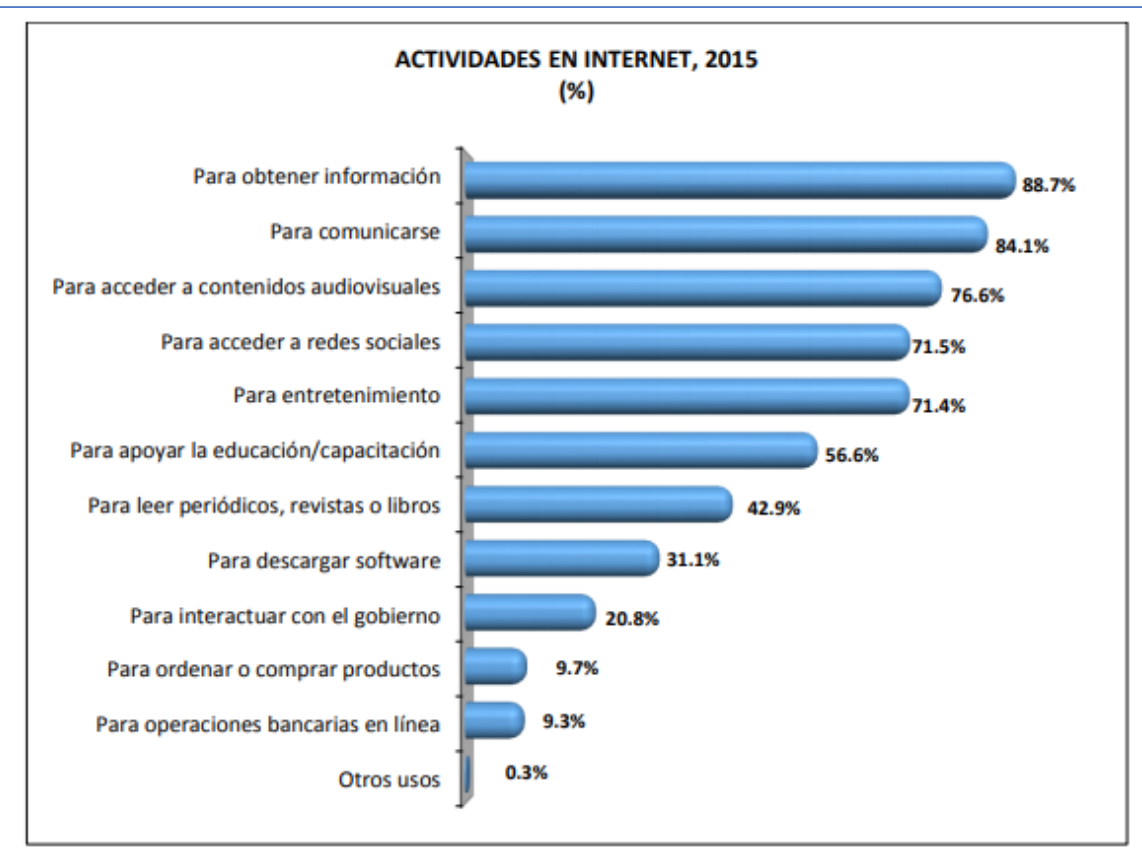

Fuente:http://www.inegi.org.mx/saladeprensa/aproposito/2016/internet2016_0. pdf

Elaborado por: Instituto Nacional de Estadística y Geografía.

La gráfica 4 muestra las principales actividades realizadas en Internet reportadas en 2015; entre las dos más recurrentes se encuentran las vinculadas a la búsqueda de información (88.7 \%) y como medio de comunicación (84.1 por ciento). Para el acceso a contenidos audiovisuales, la participación en redes sociales y como medio de entretenimiento los porcentajes se encuentran entre el 71 y el $77 \%$.

\section{Datos estadísticos}

Frecuencia del uso de internet en el Ecuador tanto adultos como los adolescentes.

Tabla N.5

\begin{tabular}{lll}
\hline Uso del internet & Frecuencia & Porcentaje \\
\hline Hombres & 36 & $36.2 \%$ \\
Jóvenes de 16-24 años & 64 & $64.9 \%$ \\
Hogares con computadoras & 24 & $24.7 \%$ \\
La provincia más & 53 & $59.1 \%$ \\
conectada : Pichincha & &
\end{tabular}

Fuente:http://dspace.ucuenca.edu.ec/bitstream/123456789/26956/1/PROYECT O\%20DE\%20INVESTIGACI\%C3\%93N.pdf

Elaborado por: Ruth Collaguazo

\section{Gráfico N.5}




\section{Uso del internet}

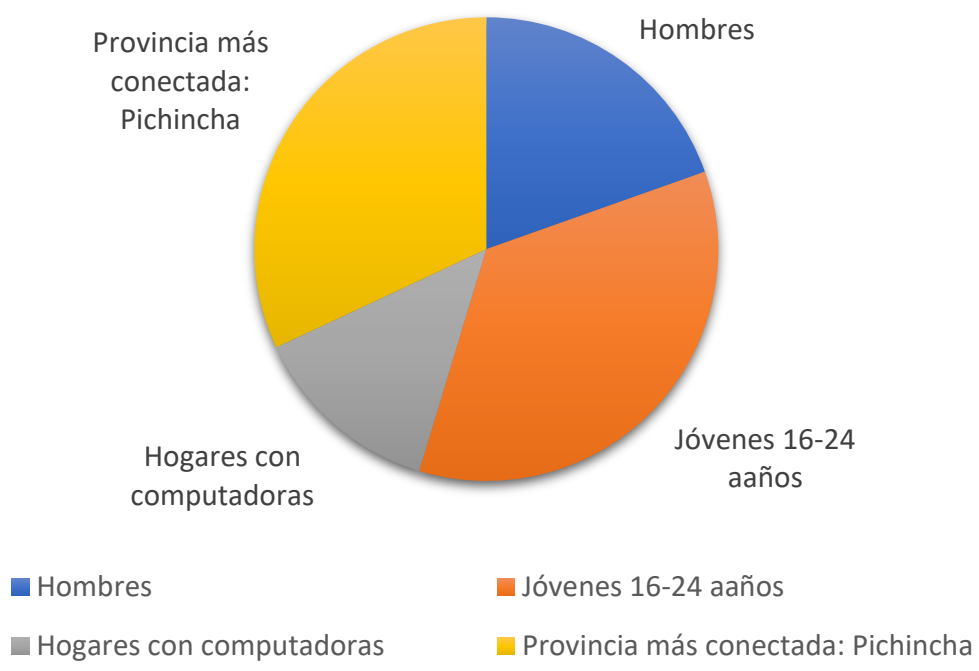

Fuente: https://www.elcomercio.com/tendencias/ecuador-internet-datostecnologia-usuarios.html

Elaborado por: El comercio

Este gráfico muestra el índice del uso del internet siendo la provincia de Pichincha la más conectada, además que los jóvenes entre los 16 y 24 año hacen más uso del internet en el Ecuador

La frecuencia del uso que hace los adolescentes de internet.

Tabla N.6

\begin{tabular}{lll}
\hline Uso del internet & Frecuencia & Porcentaje \\
\hline Conexión desde sus & 79 & $78.4 \%$ \\
hogares & & \\
Escuela & 26 & $26 \%$ \\
Biblioteca & 8 & $8 \%$ \\
Cibercafé & 5 & $5 \%$ \\
\hline
\end{tabular}

Fuente: Investigación rrealizada

Elaborado por: Daysi Vega

\section{Gráfico N.6}




\section{Usos del internet en los adolescentes}

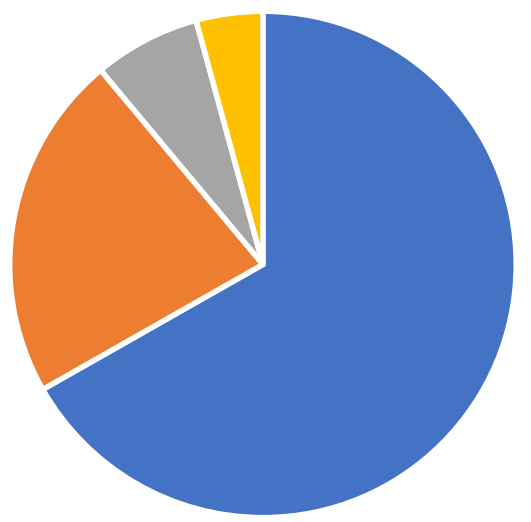

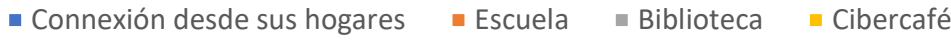

Fuente: Investigación realizada

Elaborado por: Daysi Vega

En este gráfico podemos ver que el mayor índice de conexión se más en los hogares ya que hoy en día muchos ya tienen acceso al internet con más facilidad, siendo el cibercafé con el menor índice en uso de conexión.

\section{Conclusiones}

La mayor parte del conocimiento popular sobre Internet, móvil o video juegos está basada en opiniones que reflejan los medios de comunicación.

Dada la alarma social que ha producido el mal uso de las TIC por parte de los adolescentes, se habría de facilitar la investigación sobre el uso que hacen de esto recursos.

Los adolescentes le dan un mal uso a las TIC y por lo cual se debería tomar hábitos saludables y dejar un poco esto que no se vuelva adictivo, y enfocarse en las cosas que sean favorecedoras.

$>$ Se constata la necesidad de definir criterios y diagnósticos sólidos y contrastados de la adicción a Internet, móvil y videojuegos.

\section{Referencias Bibliográficas}

Alonso Fernández, F. (2003). Las nuevas adicciones.

Black, D. W., Belsare, G., \& Schlosser, S. (1999). Clinical features, psychiatric comorbidity, and health-related quality of life in persons reporting compulsive computer use behavior. Journal of Clinical Psychiatry, 839-844.

Castellana, M. (2003). La relació de l'adolescent ambles persones significatives. 
Castellana, M. (2005). El adolescente y sus personas significativas, 18-29.

Castellana, M., Sánchez Carbonell, X., Beranuy, M., \& Graner, C. (2006). La relació de 1' adolescent amb les TIC: Un tema de rellevància social. Full Informatiu del Col·legi Oficial de Psicòlegs de Catalunya, 192-223.

Chamarro, A., \& Hernández, E. (2005). Nuevos estilos de vida en la sociedad red: una propuesta teórica enfocada a la intervención en salud. berpsicología, 2-15.

Criado, M. A. (2005). Enfermos del móvil. Recuperado el 30 de 11 de 2006, de http://www.el-mundo.es/ariadna/2005/218/1106327395.htm

Davis, R. A. (2001). A cognitive-behavioral model of pathological Internet use. Computers in Human Behavior, 187-195.

De la Gándara, J. J., \& álvarez, M. T. (2004). Patologías emergentes en salud mental. ¿Modas, enfermedades o trastornos psicosociales?, 3-15.

Echeburúa, E. (1999). ¿Adicciones sin drogas? Bilbao: Desclée de Brouwer.

Estallo, J. A. (1995). Los videojuegos. Juicios y perjuicios.

Estallo, J. A. (1995). Los videojuegos. Juicios y perjuicios.

Fiel, G. (2001). Los grupos de charla de Internet como objeto de adicción: caso IRC. Recuperado el 16 de 11 de 2006, de www.ub.edu.ar/investigaciones/tesinas/3_fiel_martinez.htm

Forunati, L., \& Magnanelly, A. M. (2002). El teléfono móvil de los jóvenes. Estudios de Juventud, 59-78.

Gee, J. P. (2004). Lo que nos enseñan los videojuegos sobre el aprendizaje y el analfabetismo.

Griffiths, M. (2000). Does Internet and computer "addiction" exist? Some case study evidence. Cyberpsychology \& Behavior, 211-218.

Griffiths, M. D. (1997). echnological addictions: Looking to the future.

Grohol, J. (2005). More spin on "Internet addiction disorder". Recuperado el 2018 de 10 de 21, de http://psychcentral.com/blog/archives/2005/04/16/internet-addictiondisorder/

Johansson, A., \& Gotestam, K. G. (2004). Internet addiction: characteristics of a questionnaire and prevalence in Norwegian youth (12-18 years). Scandinavian Journal of Psychology, 223-229. 
King, S. A. (1996). Is the Internet Addictive, or Are Addicts Using the Internet? Obtenido de http://webpages.charter.net/stormking/iad.html

Levis, D. (2002). Videojuegos: Cambios y permanencias. Comunicación y Pedagogía, 65-69.

Machargo, J., Lujan, L., León, M. D., López, P., \& Martín, M. A. (2003). Videojuegos por los adolescentes. Anuario de Filosofia, Psicologia y Sociología, 159-172.

Madrid, R. I. (2000). La adicción a Internet. Psicología Online. Recuperado el 21 de 10 de 2018, de http://www.psicologiaonline.com/colaboradores/nacho/ainternet.htm

Matute, H. (2003). Adaptarse a Internet. La coruña: La voz de Galicia.

Rodríguez, E. (2002). Jóvenes y Videojuegos: espacio, significación y conflictos. Fundación de Ayuda contra la drogadicción.

Saldaña, D. (2001). Nuevas tecnologías; nuevos instrumentos y nuevos espacios para la psicología. Apuntes de Psicología, 1, 5-10.

Sánchez Carbonell, X., Castellana, M., \& Beranuy , M. (2006). De los que padecen adicciones tecnológicas. Tutoría y orientación en la diversidad, 319-329.

Simkova, B., \& Cincera, J. (2004). Internet Addiction Disorder and Chatting in the Czech Republic. Cyberpsychology \& Behavior, 7, 536-539.

Washton, A. M., \& Boundy, D. (1991). Querer no es poder: Cómo comprender y superar las adicciones.

Wieland, D. W. (2005). Computer Addiction: Implications for nursing psychotherapy practice. Perspetive Psychiatric Care, 153-161.

Young, K. (1998). Internet Addiction. The emergence of as new clinical disorder, 1, 237244.

Young, K. S. (1999). Internet addiction: symptoms, evaluation and treatment. En L. VandeCreek y T. Jackson (Ed.), Innovations in Clinical Practice: A source book. Professional Resource Press.

\section{【Liencia}




\section{Para citar el artículo indexado.}

Velasteguí P. (2019). La influencia del internet y su impacto en los adolescentes. Revista electrónica Explorador Digital 3(1), 5-22. Recuperado desde:

http://cienciadigital.org/revistacienciadigital2/index.php/exploradordigital/article/view/342/7 54

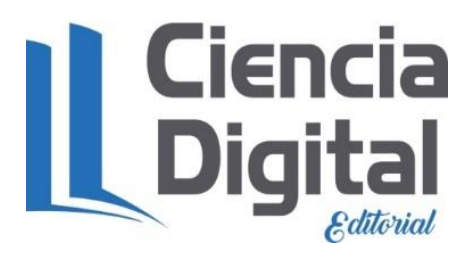

El artículo que se publica es de exclusiva responsabilidad de los autores y no necesariamente reflejan el pensamiento de la Revista Explorador Digital.

El articulo queda en propiedad de la revista y, por tanto, su publicación parcial y/o total en otro medio tiene que ser autorizado por el director o editor de la Revista Explorador Digital.
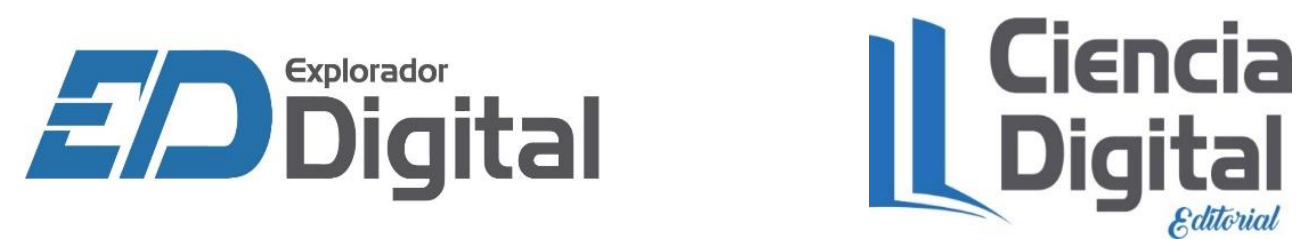